\title{
Etiological Observations in 36 Patients With Hodgkin's Lymphoma
}

\author{
WALTER WEBER and SAMUEL S. WEBER
}

Clinical Cancer Etiology Unit, Basel, Switzerland

\begin{abstract}
Background/Aim: Hodgkin's lymphoma (HL) accounts for $1 \%$ of cancers and is of largely unknown pathogenesis. This study intended to find clues about potential causes (etiology) of HL through observations on patients and their families. Patients and Methods: In 37 years of a medical oncology practice in Basel, Switzerland, 36 out of 2201 patients (1.6\%) had HL. Etiological information has been obtained from the patients with the help of a German translation of the NCI Medical History Questionnaire for Cancer Etiology, and through the study of their medical charts. Results: Findings of etiological interest were observed in the medical history of 26 out of the 36 patients with HL; these findings were grouped as follows: benign tumors (25\%), carcinogenic exposures (19\%), immunologic disorders (19\%), secondary malignancies $(14 \%)$, infections $(11 \%)$, congenital disorders (8\%) and tonsillectomies (6\%). Twenty-two out of 36 patients had one or more relatives with cancer. Conclusion: Personal and family history of patients with $H L$ is a readily available useful tool for etiology research. The fact that $H L$ is frequently associated with other cancers in patients and their relatives points to causal genetic factors.
\end{abstract}

Etiology is defined as the science concerned with the causes of a disease (1). Alert clinicians have played a key role in recognizing environmental and genetic influences in cancer etiology (2), since many carcinogens, teratogens, and genetic syndromes have been first described by alert observers (3). Hodgkin's lymphoma (HL) accounts for $1 \%$ of cancers. Most frequently two age groups are affected: patients aged between $15-35$ years, and aged $>55$ years. The probability of cure has improved dramatically over the past 50 years. HL patients survive for longer, however, with more serious and lasting damage, than almost any other group of cancer patients.

Correspondence to: Walter Weber, MD, Medical Oncology, Schützenmattstrasse 1, CH-4051 Basel, Switzerland. Tel: +41 0612617513, Fax: +41 0613119773, e-mail: cancer@bluewin.ch

Key Words: Hodgkin's lymphoma, cancer etiology.
The etiology of HL is not well understood. Environmental factors are suspected to play a role (4). Epstein-Barr virus (EBV) is detected in Hodgkin and Reed-Sternberg cells in most HL specimens from the developing world, but less often in the industrialized countries (5). Immunosuppression (HIV-infection, organ transplantation, autoimmune conditions such rheumatoid arthritis, systemic lupus erythematodes and sarcoidosis) has been shown to increase the risk for HL (5). HL shows strong familial aggregation (6). No major susceptibility genes have been identified to date. A predisposing mutation has been identified in the KDR gene (7).

\section{Patients and Methods}

Patients. In a 37-year medical oncology practice (started on April 1981), 2,201 cancer patients have been examined in hospitals affiliated to the University of Basel, Switzerland or in a private office setting (by W.W.). Out of these patients, 36 had HL. Twenty patients were female (median age at diagnosis was 29 years; range $=14-70$; median age at death or last contact was 52 years; range $=25-74$ ) and 16 were male (median age at diagnosis was 31 years; range $=15-75$; median age at death or last contact was 54 years; range $=25-76$ ).

Data collection. Etiological information has been obtained from patients with the help of a German translation of the NCI Medical History Questionnaire for Cancer Etiology (8) and through the study of patients' medical charts.

\section{Results}

Fourteen out of 36 patients have died, 8 from progressive HL, 3 from a second malignancy (1 malignant schwannoma, 1 bilateral breast cancer, and 1 carcinoma of the hypopharynx), and 3 in complete remission (1 sepsis, 1 cardiac arrhythmia, and 1 cerebrovascular insult).

Personal history. Further patient characteristics are summarized in Table I. Findings of etiological interest have been found in 26 patients (Table II). These findings can be grouped as follows: i) benign tumors occurred in 9/36 patients; ii) carcinogenic exposures have been reported by 7/36 patients; iii) immunologic disorders have been reported 
Table I. Characteristics of the 36 patients with Hodgkin's lymphoma.

\begin{tabular}{lcc}
\hline & N & N \\
\hline Gender & 16 male & 20 female \\
Stage & 3 & \\
I A & 1 & 0 \\
I B & 0 & 5 \\
II A & 0 & 3 \\
II B & 4 & 2 \\
III A & 3 & 3 \\
III B & 3 & 1 \\
IV A & 2 & 4 \\
IV B & 0 & 2 \\
Unknown & & \\
Histology & 2 & 1 \\
Lymphocyte rich & 7 & 11 \\
Nodular sclerosis & 6 & 4 \\
Mixed cellularity & 0 & 1 \\
Lymphocyte poor & 1 & 3 \\
Unknown & & \\
Treatment & 2 & 1 \\
Surgery + RT & 3 & 6 \\
Surgery + RT + CT & 2 & 0 \\
Surgery + CT & 2 & 0 \\
RT & 3 & 8 \\
RT + CT & & \\
RT + CT + Autologous bone & 1 & 0 \\
marrow transplantation & 3 & 1 \\
CT & 0 & \\
Unknown & & \\
\hline & & \\
\hline
\end{tabular}

RT, Radiotherapy; CT, chemotherapy.

by $7 / 36$ patients; iv) secondary malignant tumors occurred in $5 / 36$ patients; $v$ ) infections were reported by $4 / 36$ patients; vi) congenital disorders occurred in $3 / 36$ patients; vii) tonsillectomies were reported by $2 / 36$ patients.

Family history. Findings of etiological interest are summarized in Table III. Twenty-two patients have a positive history of cancer. Fourteen patients have one or more firstdegree relative with one or more of the following cancer types: skin $(n=3)$, leukemia $(n=3)$, breast $(n=2)$, ovary $(n=2)$ and other cancer types $(n=11)$.

\section{Discussion}

This is a retrospective study of a medical oncology practice cohort of 36 patients with HL. The main focus is laid on aspects of potential etiological interest by submitting anamnestic and clinical patient data to the scientific community for further use. The strength of such an approach is that original data are presented; however, it is only descriptive. Dr. Robert W. Miller has promoted causal
Table II. Findings of etiological interest in the personal history of 26 patients with Hodgkin's lymphoma.

\begin{tabular}{|c|c|c|}
\hline No. & Gender & Findings \\
\hline 1 & Male & $\begin{array}{l}\text { Exposure to agrochemicals. Chronic polyarthritis. } \\
\text { Interstitial lung disease under treatment. }\end{array}$ \\
\hline 2 & Male & $\begin{array}{l}\text { Exposure to dyes. Atopic dermatitis. Infectious } \\
\text { mononucleosis at the age of } 24 \text {. Chemotherapy } \\
\text { delays and compromises due to psyche. }\end{array}$ \\
\hline 3 & Male & $\begin{array}{l}\text { Bilateral cryptorchidism. Factor V-Leiden } \\
\text { (heterozygote). Combined hyperlipidemia. } \\
\text { Hypothyroidism under chemotherapy. Multiple } \\
\text { coronary artery stenoses (diagnosed at age } 45 \text { ). }\end{array}$ \\
\hline 5 & Female & Multiple allergies. \\
\hline 7 & Male & Electrician. \\
\hline 8 & Male & $\begin{array}{l}\text { Exposure to dyes. Generalized coronary heart } \\
\text { disease. Myocardial infarction at the age of } 53 \text {. } \\
\text { Sacral teratoma. }\end{array}$ \\
\hline 9 & Female & Dermoid of right ovary (D28). Achalasia (D52). \\
\hline 11 & Female & $\begin{array}{l}\text { Radiation fibrosis (D27). Fibroadenoma } \\
\text { left breast (D28). }\end{array}$ \\
\hline 12 & Female & Sarcoidosis (D68) \\
\hline 13 & Male & Malignant schwannoma paravertebral Th3 (D44). \\
\hline 14 & Female & $\begin{array}{l}\text { Cyst of the median anterior neck. Bilateral breast } \\
\text { cancer at the age of } 34 \text { (premenopausal, } \\
\text { invasive ductal, DCIS comedo type, } \\
\text { hormone receptor positive, Her } 2 \text { negative). }\end{array}$ \\
\hline 17 & Female & Recurrent pneumonias. \\
\hline 19 & Female & Borderline mucinous cystadenoma of the right ovary. \\
\hline 20 & Female & $\begin{array}{l}\text { Squamous cell carcinoma of the right hypopharynx } \\
\text { (D56). Sacral dermoid, migraine (D32). }\end{array}$ \\
\hline 21 & Female & Anorexia (D15). \\
\hline 22 & Male & $\begin{array}{l}\text { Asbestos exposure. Hypogammaglobulinemia } \\
\text { (IGM 40MG/DL N: 70-280MG/DL). }\end{array}$ \\
\hline 24 & Male & Celiac sprue (D45). \\
\hline 26 & Male & Bilateral gynecomastia since puberty. \\
\hline 28 & Female & $\begin{array}{l}\text { Infectious mononucleosis (D22). } \\
\text { Tonsillectomy (D24). Hepatitis B (D26). }\end{array}$ \\
\hline 29 & Male & $\begin{array}{l}\text { Infectious mononucleosis (D36), dental cysts } \\
\text { (D52), trichilemmoma of the scalp (D61). } \\
\text { Obesity. Coronary atherosclerosis. Diabetes type } 2 .\end{array}$ \\
\hline 30 & Female & $\begin{array}{l}\text { Cervical carcinoma in situ (D30). } \\
\text { Colorectal neoplasia (D42). }\end{array}$ \\
\hline 31 & Female & $\begin{array}{l}\text { Congenital ectopia of right kidney. } \\
\text { Radiotherapy of both breasts because of } \\
\text { keloids after bilateral mammoplasty at } \\
\text { age } 22 \text {. Worked in a solarium. }\end{array}$ \\
\hline 32 & Male & Tonsillectomy at age 39. Depressions. \\
\hline 33 & Female & $\begin{array}{l}\text { Aortic stenosis (D51). Coronary stenoses (D53). } \\
\text { Stenosis of left subclavian artery (D53). }\end{array}$ \\
\hline 34 & Male & $\begin{array}{l}\text { Squamous cell skin carcinoma (D55). Atopic } \\
\text { dermatitis in childhood. Psychic problems. }\end{array}$ \\
\hline 35 & Male & $\begin{array}{l}\text { Generalized lymphadenopathy (D29) } \\
\text { lasting for } 3 \text { years. }\end{array}$ \\
\hline
\end{tabular}

Insulator age 16-27 (asbestos exposure). Zoo keeper age 27-63. Death at the age of 66 due to occlusions of left carotid artery and left posterior cerebral artery. 
Table III. Findings of etiological interest in the family history of 22 (61\%) patients with Hodgkin's lymphoma.

\begin{tabular}{|c|c|c|}
\hline No. & Gender & Findings \\
\hline 2 & M & $\begin{array}{l}\text { Leukemia }\left(\mathrm{F}, 2^{\circ} \mathrm{fs},+1, \mathrm{nv}\right) . \text { Brain mal }\left(\mathrm{M}, 2^{\circ} \mathrm{fs},+2, \mathrm{nv}\right) \text {. Colorectal } \mathrm{mal}\left(\mathrm{F}, 2^{\circ} \mathrm{ms}, \mathrm{nv}\right) \text {. } \\
\text { Colorectal mal }\left(\mathrm{F}, 3^{\circ} \mathrm{ms}, \mathrm{nv}\right) \text {. Atopic dermatitis in } 5 \text { relatives. }\end{array}$ \\
\hline 3 & M & Rectal ca $\left(\mathrm{M}, 1^{\circ} \mathrm{fs}, \mathrm{D} 69,+, \mathrm{v}\right)$. Rheumatoid arthritis (f, $\left.1^{\circ} \mathrm{ms}, \mathrm{D} 51,86\right)$ \\
\hline 5 & $\mathrm{~F}$ & $\begin{array}{l}\text { Prostate ca D68 + CML D72 + Squamous cell skin ca D76 (M, } 1^{\circ} \mathrm{fs}, 81 \text {, v). Prostate mal + } \\
\text { chronic leukemia (M, } 2^{\circ} \mathrm{fs},+91 \text {, nv). Prostate mal }\left(\mathrm{M}, 2^{\circ} \mathrm{ms}, \mathrm{D} 65,+86, \mathrm{nv}\right) . \text { OMF }\left(\mathrm{F}, 2^{\circ} \mathrm{ms}, \mathrm{D} 68,+76, \mathrm{nv}\right) \text {. } \\
\text { OMF (F, } 2^{\circ} \mathrm{ms}, \mathrm{D} 71 \text {, v). Lung mal }\left(\mathrm{F}, 2^{\circ} \mathrm{fs}, \mathrm{D} 68,+ \text {, nv). GI mal }\left(\mathrm{M}, 2^{\circ} \mathrm{fs}, \mathrm{D} 67, \mathrm{nv}\right) \text {. GI mal }\left(\mathrm{F}, 3^{\circ} \mathrm{fs},+, \mathrm{nv}\right)\right.\end{array}$ \\
\hline 6 & $\mathrm{~F}$ & Stomach mal $\left(\mathrm{M}, 1^{\circ} \mathrm{fs},+60, \mathrm{nv}\right)$. Stomach mal $\left(\mathrm{M}, 2^{\circ} \mathrm{fs},+60, \mathrm{nv}\right)$ \\
\hline 10 & M & Lung mal $\left(\mathrm{M}, 2^{\circ} \mathrm{fs},+60, \mathrm{nv}\right)$ \\
\hline 11 & $\mathrm{~F}$ & Stomach mal $\left(\mathrm{M}, 2^{\circ} \mathrm{fs},+50, \mathrm{nv}\right)$. Stomach mal (f, $\left.2^{\circ} \mathrm{fs},+60, \mathrm{nv}\right)$ \\
\hline 12 & $\mathrm{~F}$ & $\begin{array}{l}\text { Ovarian ca endometroid type }+ \text { congenital bronchiectases }+ \text { Factor V-Leiden }\left(F, 1^{\circ} \text { daughter, D } 34,66 \text {, }\right. \\
\text { v). Ovarian ca }\left(F, 1^{\circ} \text { mother, }+74, \mathrm{nv}\right) \text {. Gyn mal }+ \text { skin mal }\left(\mathrm{F}, 1^{\circ} \text { daughter, } \mathrm{D} 43, \mathrm{nv}\right)\end{array}$ \\
\hline 13 & $\mathrm{~F}$ & GI mal $\left(\mathrm{F}, 2^{\circ} \mathrm{ms},+62\right.$, nv). Lung mal $\left(\mathrm{M}, 2^{\circ} \mathrm{ms},+69\right.$, nv). Kidney mal $\left(\mathrm{F}, 3^{\circ} \mathrm{ms},+72\right.$, nv). GI mal $\left(\mathrm{F}, 3^{\circ} \mathrm{ms},+73\right.$, nv). \\
\hline 14 & $\mathrm{~F}$ & Testicular ma $\left(\mathrm{M}, 1^{\circ} \mathrm{fs}, \mathrm{D} 60, \mathrm{nv}\right)$. Testicular mal $\left(\mathrm{M}, 2^{\circ} \mathrm{fs},+75, \mathrm{nv}\right)$. Leukemia $\left(\mathrm{M}, 3^{\circ} \mathrm{ms},+45, \mathrm{nv}\right)$ \\
\hline 17 & $\mathrm{~F}$ & Bone marrow mal $\left(\mathrm{M}, 3^{\circ} \mathrm{ms},+43, \mathrm{nv}\right)$. Stomach mal $\left(\mathrm{F}, 2^{\circ} \mathrm{ms},+70, \mathrm{nv}\right)$ \\
\hline 18 & $\mathrm{~F}$ & Brain lesion $\left(1^{\circ}, \mathrm{D} 32, \mathrm{nv}\right)$. GI mal $\left(\mathrm{F}, 2^{\circ} \mathrm{fs}, \mathrm{nv}\right)$. Multiple sclerosis $\left(\mathrm{M}, 1^{\circ} \mathrm{fs}, \mathrm{D} 41, \mathrm{nv}\right)$ \\
\hline 20 & $\mathrm{~F}$ & $\begin{array}{l}\text { Skin mal }\left(\mathrm{F}, 2^{\circ} \mathrm{fs}, \mathrm{nv}\right) \text {. Lung mal }\left(\mathrm{M}, 2^{\circ} \mathrm{fs},+57 \text {, nv). Lung mal }\left(\mathrm{M}, 2^{\circ} \mathrm{fs},+, \mathrm{nv}\right) \text {. Gyn mal }\left(\mathrm{F}, 2^{\circ} \mathrm{ms},+, \mathrm{nv}\right) \text {. }\right. \\
\text { Malformations }\left(\mathrm{F}, 2^{\circ} \mathrm{ms},+1\right) \text {. }\end{array}$ \\
\hline 21 & $\mathrm{~F}$ & $\begin{array}{l}\text { Chronic myelo-monocytic leukemia }\left(\mathrm{F}, 1^{\circ} \mathrm{ms}, \mathrm{D} 63,+65 \text {, v). Leukemia }\left(\mathrm{F}, 3^{\circ} \mathrm{fs},+40 \text {, nv). }\right.\right. \\
\text { Lung mal }\left(\mathrm{M}, 2^{\circ} \mathrm{fs},+ \text {, nv). Suicide }\left(\mathrm{M}, 1^{\circ},+25\right) \text { Neurodermitis }\left(\mathrm{F}, 1^{\circ}, \mathrm{D} 2\right) \text {. Pernicious anemia }\left(\mathrm{M}, 1^{\circ} \mathrm{fs}, \mathrm{D} 36,+78\right) \text {. }\right.\end{array}$ \\
\hline 24 & M & Gyn. mal $\left(\mathrm{F}, 1^{\circ} \mathrm{ms}, \mathrm{nv}\right)$ \\
\hline 27 & M & GI mal (F, $\left.1^{\circ} \mathrm{ms}, \mathrm{D} 59,63, \mathrm{nv}\right)$. Unknown primary mal $\left(\mathrm{M}, 2^{\circ} \mathrm{fs}, \mathrm{D} 55, \mathrm{nv}\right)$. \\
\hline 28 & $\mathrm{~F}$ & Unknown primary mal $\left(\mathrm{M}, 2^{\circ} \mathrm{ms},+80, \mathrm{nv}\right)$ \\
\hline 29 & M & $\begin{array}{l}\text { Breast ca, ductulolobular, left side, } \mathrm{ER}^{+} \mathrm{PR}^{+} \mathrm{HER} 2^{-}\left(\mathrm{F}, 1^{\circ}, \mathrm{D} 64,76, \mathrm{v}\right) \text {. Spindle cell lipoma of } \\
\text { the skin }\left(\mathrm{M}, 1^{\circ}, \mathrm{D} 75,79, \mathrm{v}\right) \text {. Squamous cell ca of the tongue }\left(\mathrm{M}, 1^{\circ} \mathrm{fs}, \mathrm{D} 58,+61 \text {, }\right. \\
\mathrm{v}) \text {. Endometrial ca }\left(\mathrm{F}, 1^{\circ} \mathrm{ms}, \mathrm{D} 60,+81 \text {, v). Uterine mal. }\left(\mathrm{F}, 2^{\circ} \mathrm{ms},+50, \mathrm{nv}\right) \text {. Breast mal }\left(\mathrm{F}, 3^{\circ} \mathrm{ms}, \mathrm{nv}\right) \text {. }\right. \\
\text { Larynx mal. }\left(\mathrm{M}, 3^{\circ} \mathrm{ms},+63, \mathrm{nv}\right) \text {. Obesity of } 2 \text { sisters, } 1 \text { brother and both parents. }\end{array}$ \\
\hline 31 & $\mathrm{~F}$ & Stomach mal $\left(\mathrm{M}, 2^{\circ} \mathrm{ms},+, \mathrm{nv}\right)$. Liver mal $\left(\mathrm{M}, 2^{\circ} \mathrm{ms},+, \mathrm{nv}\right)$ \\
\hline 33 & $\mathrm{~F}$ & Leukemia $\left(\mathrm{M}, 1^{\circ} \mathrm{fs},+82, \mathrm{nv}\right)$ \\
\hline 34 & M & Cutaneous $\mathrm{T}$ cell lymphoma $\left(\mathrm{M}, 2^{\circ}, \mathrm{D} 14, \mathrm{nv}\right) . \mathrm{NHL}\left(\mathrm{M}, 1^{\circ} \mathrm{fs}, \mathrm{D} 75,+, \mathrm{nv}\right)$ \\
\hline 35 & M & $\begin{array}{l}\text { Breast ca }\left(\mathrm{F}, 1^{\circ} \mathrm{ms}, \mathrm{D} 65,+81, \mathrm{v}\right) \text {. Prostate mal }\left(\mathrm{M}, 2^{\circ} \mathrm{ms}, \mathrm{D} 67,+71, \mathrm{nv}\right) \text {. Atrial myxoma }\left(\mathrm{F}, 1^{\circ},+19, \mathrm{nv}\right) \text {. } \\
\text { 2nd wife: FH suggestive of Lynch syndrome. }\end{array}$ \\
\hline 36 & $\mathrm{~F}$ & Stomach mal $\left(\mathrm{F}, 2^{\circ} \mathrm{fs},+70, \mathrm{nv}\right)$ \\
\hline
\end{tabular}

ca, Carcinoma; D, diagnosis; D69, age at diagnosis (69 years); ER ${ }^{+}$, estrogen receptor positive; F, female; FH, family history; Fs, father's side; GI, gastrointestinal; Gyn, gynecological; HER2-, herceptin negative; m, male; mal, malignancy; ms, mother's side; NHL, non-hodgkin lymphoma; nv, not verified; OMF, osteomyelofibrosis; $\mathrm{PR}^{+}$, progesteron receptor positive; syndr, syndrome; v, verified by autopsy/cytology/histology report; $1^{\circ}$, first degree relative; $2^{\circ}$, second degree relative; $3^{\circ}$, third degree relative; + , dead; +1 , age at death (1 year); 86 , actual age $(86$ years).

research by alert clinicians (3). A comprehensive review of already published data would be beyond the scope of such a small study.

The personal history of 26 out of the 36 HL patients revealed aspects of potential causal relevance, such as benign and malignant tumors, carcinogenic exposures; immunologic, infectious and congenital disorders. Out of $36 \mathrm{HL}$ patients, 22 had one or more close relative with malignancies. This finding supports the conclusion of a previous report, which demonstrated that familial cancers might have a genetic basis and highlighted the importance of recording family history and survival data (9). Indeed, although HL patients survive for longer than almost any other group of cancer patients, studies including old ( $>65$ years old) and long-term ( $>5$ years) survivors have lagged behind (10).

Patient registries and biobanking are increasingly recognized as crucial tools for rare disease research (11). Therefore, in this study, sera from $19 \mathrm{HL}$ patients have been stored for further genetic analysis studies.

In conclusion, a detailed presentation of anamnestic and clinical data of HL patients combined with the preservation of biological material for future laboratory studies could facilitate the identification of etiological (causal) factors of the disease. 


\section{Conflicts of Interest}

The Authors declare no conflicts of interest.

\section{Authors' Contributions}

Conception and design of the study, provision of study materials or patients, data analysis and interpretation, as well as final approval of manuscript were done by Walter Weber. Both Walter Weber and Samuel S. Weber contributed to the financial support, collection and assembly of data, and writing of the manuscript.

\section{References}

1 Karp I and Miettinen OS: On the essentials of etiologic research for preventive medicine. Eur J Epidemiol 29: 455-457, 2014. PMID: 24952165. DOI: 10.1007/s10654-014-9928-x

2 Miller RW: Rare events as clues to cancer etiology: the eighteenth annual Symposium of the Princess Takamatsu Cancer Research Fund. Cancer Res 48: 3544-3548, 1988.

3 Miller RW: Living history autobiography: clinical genetics: key to cancer etiology. Am J Med Genet 76: 9-20, 1998. PMID: 9508058. DOI: 10.1002/(sici)1096-8628(19980226)761<9 ::aidajmg2>3.0.co:2-o

4 Grufferman S and Delzell E: Epidemiology of Hodgkin's disease. Epidemiol Rev 6: 76-106, 1984. PMID: 6092122. DOI: 10.1093/oxfordjournals.epirev.a036276

5 Shanbhag S and Ambinder R: Hodgkin Lymphoma: a review and update on recent progress. CA Cancer J Clin 68: 116-132, 2018. PMID: 29194581. DOI: 10.3322/caac. 21438

6 Kharazmi E, Fallah M, Pukkala E, Olsen JH, Tryggvadottir L, Sundquist K, Tretli S and Hemminki K: Risk of familial classical Hodgkin lymphoma by relationship, histology, age, and sex: a joint study from five Nordic countries. Blood 126: 19901995, 2015. PMID: 26311361. DOI: 10.1182/blood-2015-04639781
7 Rotunno M, McMaster ML, Boland J, Bass S, Zhang X, Burdett L, Hicks B, Ravichandran S, Luke BT, Yeager M, Fontaine L, Hyland PL, Goldstein AM, NCI DCEG Cancer Sequencing Working Group, NCI DCEG Cancer Genomics Research Laboratory, Chanok SJ, Caporaso NE, Tucker MA and Goldin LR: Whole exome sequencing in families at high risk for Hodgkin lymphoma: identification of a predisposing mutation in the KDR gene. Haematologica 101: 853-860, 2016. PMID: 27365461. DOI: 10.3324/haematol.2015.135475

8 Appendix. In: Genetics of human cancer. JJ Mulvihill, RW Miller and JF Fraumeni, Jr. (eds). Raven Press, New York, pp. 489-493, 1977.

9 Hemminki K, Sundquist J and Brandt A: Familial mortality and familial incidence in cancer. J Clin Oncol 29: 712-718, 2011. PMID: 21205747. DOI: 10.1200/JCO.2010.30.5664

10 Rowland JH, Gallicchio L, Mollica M, Saiontz N, Falisi AL and Tesauro G: Survivorship science at the NIH: Lessons learned from grants funded in fiscal year 2016. J Natl Cancer Inst 111: 109-117, 2019. PMID: 30657942. DOI : 10.1093/jnci/div208

11 Lochmüller H, Torrent i Farnell J, Le Cam Y, Jonker AH, Lau LPL, Baynam KM, Kaufmann P, Dawkins HJS, Lasko P, Austin $\mathrm{CP}$ and Boykott KM on behalf of the IRDiRC Consortium Assembly: The International Rare Diseases Research Consortium: Policies and Guidelines to maximize impact. Eur J Hum Genet 25: 1293-1302, 2017. PMID: 29158551. DOI: 10.1038/s41431017-0008-Z
Received July 6, 2019

Revised October 21, 2019 Accepted November 12, 2019 